\title{
HERMITIAN SURFACES OF CONSTANT HOLOMORPHIC SECTIONAL CURVATURE II
}

\author{
TAKUJI SATO AND KOUEI SEKIGAWA
}

\begin{abstract}
A bstract. The present paper is a continuation of our previous work [7]. We shall prove that a copmact Hermitian surface of pointwise positive constant holomorphic sectional curvature is biholomorphically equivalent to a. complex projective surface.
\end{abstract}

\section{\$1. Introduction}

An almost Hermitian manifold with integrable almost complex structure is called a Hermitian manifold. The holomorphic sectional curvature $\mathbb{H}$ of a Hermitian manifold $M=(M, J, g)$ can be regarded as a differentiable function on the unit tangent bundle $U(M)$ of $M$. A Hermitian manifold $M=(M, J, g)$ is said to be of pointwise constant holomorphic sectional curvature if the function $H$ is constant along each fibre of $U(M)$. Especially, $M$ is said to be of constant holomorphic sectional curvature if $\mathbb{H}$ is constant on the whole of $U(M)$. In the present paper, we shall deal only with the curvature tensor with respect to the Riemannian connection of $M$.

In the previous article [7], we have proved the following

Theorem $\mathrm{A}$. Let $M=(M, J, g)$ be a compact Hermitian surface of nonpositive constant holomorphic sectional curvature. Then $M$ is a Kähler surface.

Theorem B. Let $M=(M, J, g)$ be a compact Hermitian surface of positive

Received September 15, 1991. 
constant holomorphic sectional curvature. Then the Euler number $\chi(M)$ and the Chern number $c_{1}(M)^{2}$ are positive, and the Pontrjagin number $p_{1}(M)$ is nonnegative (and hence, $M$ is an algebraic surface with positive Euler number and non-negative signature).

We have also proved that the Pontrjagin number $p_{1}(M)$ of a compact Hermitian surface $M=(M, J, g)$ of pointwise constant holomorphic sectional curvature is non-negative, and $p_{1}(M)$ is equal to zero if and only if $M$ is a locally conformal Kähler surface with $\tau=3 \tau^{*}$. The purpose of the present paper is to improve the above Theorem B. Namely, we shall prove the following

Theorem C. Let $M=(M, J, g)$ be a compact Hermitian surface of pointwise positive constant holomorphic sectional curvature. Then $M$ is biholomorphically equivalent to a complex projective surface $\mathbb{P}^{2}(\mathbb{C})$.

We adopt the same notational convention as in our previous paper [7].

\section{§2. Preliminaries}

Let $M=(M, J, g)$ be a Hermitian surface and $\Omega=\left(\Omega_{i j}\right)$ the Kähler form of $M$ defined by $\Omega_{i j}=g_{i k} J_{j}^{k}$. We denote by $\nabla, \mathbb{R}=\left(\mathbb{R}_{i j k} l\right), \rho=\left(\rho_{i j}\right)$ and $\tau$, the Riemannian connection, the Riemannian curvature tensor, the Ricci tensor and the scalar curvature of $M$, respectively. The Ricci $*$-tensor $\rho^{*}=\left(\rho_{i j}^{*}\right)$ and the $*$-scallar curvature $\tau^{*}$ are given respectively by

$$
\begin{aligned}
\rho_{i j}^{*} & =\frac{1}{2} J_{j}^{s} R_{i s a}^{b} J_{b}^{a}, \\
\tau^{*} & =g^{i j} \rho_{i j}^{*} .
\end{aligned}
$$

We denote by $\omega=\left(\omega_{i}\right)$ the Lee form of $M$. The Lee form $\omega$ is given by $\omega=\delta \Omega \circ J$ and satisfies the following equalities:

$$
\begin{aligned}
J^{i j} \nabla_{i} \omega_{j} & =0, \\
2 \nabla_{i} J_{j}^{k} & =\omega_{a} J_{j}^{a} \delta_{i}^{k}-\omega_{a} J^{k a} g_{i j}-\omega_{j} J_{i}^{k}+\omega^{k} J_{i j}, \\
\tau-\tau^{*} & =2 \delta \omega+\|\omega\|^{2},
\end{aligned}
$$


([7], [10]).

We denote by $\chi(M), c_{1}(M)^{2}, c_{2}(M)$ and $p_{1}(M)$ the Euler number, the first Chern number, the second Chern number and the first Pontrjagin number of $M$, respectively. We note that $c_{2}(M)$ is equal to $\chi(M)$, and $\frac{1}{3} p_{1}(M)$ is equal to the Hirzebruch signature of $M$.

Now, let $\tilde{\nabla}$ be the Hermitian connection (known also as the Chern connection) of $M$ and $\widetilde{\Gamma}=\left(\widetilde{\Gamma}_{j k}^{i}\right)$ the coefficients of the connection $\tilde{\nabla}$ in each coordinate neighborhood of $M$. Then we have

$$
\widetilde{\Gamma}_{j k}^{i}=\Gamma_{j k}^{i}-\frac{1}{2} \omega_{k} \delta_{j}^{i}-\frac{1}{2} J_{j}^{a} \omega_{a} J_{k}^{i}+\frac{1}{2} g_{j k} \omega^{i},
$$

where $\Gamma=\left(\Gamma_{j k}^{i}\right)$ are the coefficients of the Riemannian connection $\nabla$ (cf. [10]). By (2.6), the curvature tensor $\widetilde{R}=\left(\widetilde{R}_{i j k} l\right)$ of the connection $\widetilde{\nabla}$ is given by

$$
\begin{aligned}
\widetilde{\mathbb{R}}_{i j k^{l}}= & \mathbb{R}_{i j k^{l}}+\frac{1}{2}\left\{\left(\nabla_{j} \omega_{k}+\frac{1}{2} \omega_{j} \omega_{k}\right) \delta_{i}^{l}-\left(\nabla_{i} \omega_{k}+\frac{1}{2} \omega_{i} \omega_{k}\right) \delta_{j}^{l}\right. \\
& \left.+\left(\nabla_{i} \omega^{l}+\frac{1}{2} \omega_{i} \omega^{l}\right) g_{j k}-\left(\nabla_{j} \omega^{l}+\frac{1}{2} \omega_{j} \omega^{l}\right) g_{i k}\right\} \\
& +\frac{\|\omega\|^{2}}{4}\left(g_{i k} \delta_{j}^{l}-g_{j k} \delta_{i}^{l}-2 J_{i j} J_{k}^{l}\right) \\
& +\frac{1}{2} J_{k}^{l}\left\{J_{i}^{a}\left(\nabla_{j} \omega_{a}+\omega_{j} \omega_{a}\right)-J_{j}^{a}\left(\nabla_{i} \omega_{a}+\omega_{i} \omega_{a}\right)\right\} .
\end{aligned}
$$

We denote by $\tilde{\rho}=\left(\tilde{\rho}_{i j}\right)$ and $\tilde{\rho}^{*}=\left(\widetilde{\rho}_{i j}^{*}\right)$ the tensor fields on $M$ of type $(0,2)$ defined respectively by

and.

$$
\tilde{\rho}_{i j}=\widetilde{R}_{k i j k}
$$

$$
\widetilde{\rho}_{i j}^{*}=\frac{1}{2} J_{j}^{s} \widetilde{R}_{i s a} b J_{b}^{a} .
$$

Furthermore, we put

$$
\tilde{\tau}=g^{i j} \tilde{\rho}_{i j} \quad \text { and } \quad \tilde{\tau}^{*}=g^{i j} \tilde{\rho}_{i j}^{*} \text {. }
$$


Then, by (2.7), we have easily

$$
\begin{aligned}
\tilde{\rho}_{j k}= & \rho_{j k}+\frac{1}{2}\left\{\nabla_{j} \omega_{k}-J_{j}^{a} J_{k}^{b}\left(\nabla_{b} \omega_{a}+\omega_{b} \omega_{a}\right)-(\delta \omega) g_{j k}\right\}, \\
\tilde{\rho}_{j k}^{*}= & \rho_{j k}^{*}-\frac{1}{2}\left(\nabla_{j} \omega_{k}+J_{j}^{a} J_{k}^{b} \nabla_{b} \omega_{a}\right) \\
& -\frac{3}{4}\left(\omega_{j} \omega_{k}+J_{j}^{a} J_{k}^{b} \omega_{b} \omega_{a}-\|\omega\|^{2} g_{j k}\right) .
\end{aligned}
$$

From (2.5), (2.8) and (2.9), we have

$$
\begin{aligned}
\widetilde{\tau} & =\tau^{*}+\frac{1}{2}\|\omega\|^{2}, \\
\widetilde{\tau}^{*} & =\frac{1}{2}\left(\tau+\tau^{*}\right)+\|\omega\|^{2} .
\end{aligned}
$$

Let $u$ and $v$ be the two scalar curvatures of Hermitian geometry introduced in the work of $A$. Balas [3]. Then we have

$$
\tilde{\tau}=2 v \text { and } \quad \tilde{\tau}^{*}=2 u \text {. }
$$

We now assume that $M$ is of pointwise constant holomorphic sectional curvature $c=c(p)$. Then we have

$$
\tau+3 \tau^{*}=24 c
$$

(cf. [7]).

\section{§3. Proof of Theorem C}

Let $M=(M, J, g)$ be a compact Hermitian surface of pointwise positive constant holomorphic sectional curvature. Then the characteristic numbers $\chi(M)$, $c_{1}(M)^{2}$ and $p_{1}(M)$ are given respectively by

$$
\begin{aligned}
\chi(M) & =\frac{1}{32 \pi^{2}} \int_{M}\left\{12 c^{2}-\frac{1}{16}\left(\tau-\tau^{*}\right)^{2}+\frac{1}{2} \tau^{*}\|\omega\|^{2}\right\} d M, \\
c_{1}(M)^{2} & =\frac{1}{32 \pi^{2}} \int_{M}\left\{\left(\tau^{*}\right)^{2}+\tau^{*}\|\omega\|^{2}+\|d \omega\|^{2}\right\} d M, \\
p_{1}(M) & =\frac{1}{32 \pi^{2}} \int_{M}\left\{\frac{1}{12}\left(\tau-3 \tau^{*}\right)^{2}+\|d \omega\|^{2}\right\} d M,
\end{aligned}
$$


(cf. [7]).

Now, by (2.10), (2.12) and (2.13), we have

$$
\begin{aligned}
u+v & =\frac{1}{2}\left(\widetilde{\tau}^{*}+\tilde{\tau}\right)=\frac{1}{4}\left(\tau+3 \tau^{*}\right)+\frac{3}{4}\|\omega\|^{2} \\
& =6 c+\frac{3}{4}\|\omega\|^{2}>0 .
\end{aligned}
$$

Thus, from (3.4), taking account of the result of $A$. Balas ([3], Theorem 1), we see that the plurigenera of $M$ all vanish, that is, the Kodaira dimension of $M$ is equal to -1 . Thus, the Noether formula ([5]) is of the form

$$
c_{1}(M)^{2}+c_{2}(M)=12(1-q), \quad(q \geq 0),
$$

where $q=q(M)$ is the irregularity of $M$. From (3.5), taking account of Theorem $\mathbb{B}$, we have $q=0$. Thus, (3.5) reduces to

$$
c_{1}(M)^{2}+c_{2}(M)=12 .
$$

Referring to the well-known classification of compact complex surfaces (see, for example, [4], p. 415), we infer that $M$ is rational, equivalently, obtained by successive blowing up's from a complex projective surface $P^{2}(\mathbb{C})$ or a (geometrically) ruled surface over a complex projective line $\mathbb{P}^{1}(\mathbb{C})$.

Since $c_{2}(M)=\chi(M)>0$ by Theorem $B$, Miyaoka's inequality is of the form

$$
c_{1}(M)^{2} \leq 3 c_{2}(M)
$$

([5], [6]). By (3.6) and (3.7), we have

$$
c_{2}(M) \geq 3
$$

Furthermore, by the Wu's theorem ([11], Theorem 10, p. 74) and Theorem B, we have

$$
c_{1}(M)^{2} \geq 2 c_{2}(M)
$$

So, by (3.6), (3.8) and (3.9), we have

$$
c_{2}(M)=3 \text { or } 4 \text {. }
$$


We assume that $c_{2}(M)=4$. Then, by (3.6), we get $c_{1}(M)^{2}=8$. Thus, from the Wu's theorem, it follows immediately that $p_{1}(M)=0$, and hence $M$ is locally conformal $\mathbb{K} a ̈ h l e r$ surface with $\tau=3 \tau^{*}$ by virtue of (3.3). Then, by (2.13), we have

$$
\tau=12 c>\tau^{*}=4 c .
$$

Since $M$ is simply connected, $M$ is a globally conformal $\mathbb{K}$ ähler surface. Thus, there exists a differentiable function $f$ on $M$ such that $\omega=d f$. Then the equality (2.5) reduces to

$$
\tau-\tau^{*}=-2 \Delta f+\|\operatorname{grad} f\|^{2},
$$

where $\Delta=-\delta d$ is the Laplace-Beltrami operator acting on differentiable functions on $M$. Let $p_{0}$ be a point of $M$ such that $f\left(p_{0}\right)=\operatorname{Min}_{p \in M} f(p)$. Then, by (3.11), we see that $\tau \leq \tau^{*}$ at $p_{0}$. But this is a contradiction, and hence $c_{2}(M)=\chi(M)=3$.

Thus, by (3.6) and the Wu's theorem, we have

$$
c_{1}(M)^{2}=9 \text { and } \quad p_{1}(M)=3 .
$$

The second Betti number $b_{2}(M)$ of $M$ is written as usual by $b_{2}(M)=b_{+}+b_{-}$, and the signature of $M\left(=\frac{1}{3} p_{1}(M)\right)$ is equal to $b_{+}-b_{-}$. Since $\chi(M)=2+b_{2}(M)=$ $2+b_{+}+b_{-}$and $b_{+}-b_{-}=1$ by (3.12), we have

$$
b_{+}=1, \quad b_{-}=0 .
$$

Summing up the above arguments, we can conclude that $M$ is biholomorphically equivalent to a complex projective surface $\mathbb{P}^{2}(\mathbb{C})$. This completes the proof of Theorem C.

\section{References}

[1] Balas, A., "Compact Hermitian manifolds of constant holomorphic sectional curvature", Math. Z. 189, 193-210, (1985).

[2] Balas, A. and Gauduchon, P., "Any Hermitian metric of constant non-positive (Hermitian) holomorphic sectional curvature on a compact complex surface is Kähler", Math. Z. 190, 39-43, (1985). 
[3] Baslas, A., "On the sum of the Hermitian scalar curvatures of a compact Hermitian manifold", Math. Z. 195, 429-432, (1987).

[4] Bombieri, E. and Husemoller, D., "Classification and embeddings of surfaces", Proc. Symp. Pure Math. 29, 329-420, (1975).

[5] Kodaira, $\mathbb{K}$., "On the structure of compact analytic surfaces I, II, III, IV", Amer. J. Math. 86, 88, 90, 751-798, 682-721, 55-83, 1048-1066, (1964; 1966; 1968).

[6] Miyaoka, Y., "On the Cherm numbers of surfaces of general type", Invent. Math. 42, 225-237, (1977).

[7] Sato, T. and Sekigawa, K., "Hermitian surfaces of constant holomorphic sectional curvature", Math. 2. 205, 659-668, (1990).

[8] Sekigawa, K., "On some 4-dimensional compact almost Hermitian manifolds", J. Ramanujan Math. Soc. 2, 101-116, (1987).

[9] Tricerri, F. and Vaisman, I., "On some 2-dimensional Hermitian manifolds", Math. Z. 192, 205-216, (1986).

[10] Vaisman, I., "Some curvature properties of complex surfaces", Ann. Mat. Pura Appl. 32, 1-18, (1982).

[11] Wu, W. T., "Sur les classes caractéristiques des structures fibrées sphériques", Actral. Sci. Ind. 1183, 1-89, (1952).

Faculty of Technology, Kanazawa University, Kanazawa 920, Japan.

Department of Mathematics, Faculty of Science, Niigata University, Niigata 950-21, Japan. 